\title{
ADAPTAÇÃO TRANSCULTURAL DO QUESTIONÁRIO THREE DAY PHYSICAL ACTIVITY RECALL
}

CROSS-CULTURAL ADAPTATION OFTHE QUESTIONNAIRE THREEDAY PHYSICAL ACTIVITY RECALL

ADAPTACIÓN TRANSCULTURAL DEL CUESTIONARIO THREE DAY PHYSICAL ACTIVITY RECALL

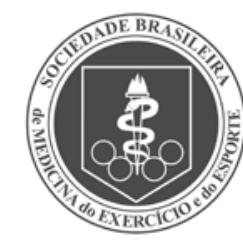

Artigo Original

Original ARTICLE

Artículo Original

\author{
Vinicius de Oliveira Damasceno' \\ (Profissional de Educação Física) \\ André Calil e Silva ${ }^{2}$ \\ (Profissional de Educação Física) \\ Paulo Roberto Amorim ${ }^{3}$ \\ (Profissional de Educação Física) \\ Jorge Roberto Perrout de Lima² \\ (Profissional de Educação Física) \\ Jeferson Macedo Vianna ${ }^{2}$ \\ (Profissional de Educação Física) \\ Reginaldo Gonçalves ${ }^{4}$ \\ (Profissional de Educação Física) \\ Francisco Zacaron Werneck ${ }^{5}$ \\ (Profissional de Educação Física) \\ Joel Alves Lamounier ${ }^{6}$ \\ (Médico Pediatra)
}

1. Universidade Federal de Pernambuco (UFPE), Recife, PE, Brasil. 2. Universidade Federal de Juiz de Fora (UFJF), Juiz de Fora, MG, Brasil. 3. Universidade Federal de Viçosa (UFV), Viçosa, MG, Brasil.

4. Universidade Federal de Belo Horizonte (UFMG), Belo Horizonte, MG, Brasil.

5. Universidade Federal de Ouro Preto (UFOP), Ouro Preto, MG, Brasil. 6. Universidade Federal de São João Del Rei (UFSJ), São João Del Rei, $M G$, Brasil.

\section{Correspondência:}

Av. Prof. Moraes Rego, 1235, Cidade Universitária, Departamento de Educação Física - UFPE, Recife, PE, Brasil. 50670-901.

vinicius.damasceno@gmail.com

\section{RESUMO}

Introdução: Diversos são os instrumentos que mensuram atividade física no Brasil. Um deles é o Three Day Physical Activity Recall, porém, até o momento, nenhuma ferramenta foi submetida à adaptação transcultural. Objetivo: Descrever os procedimentos e critérios de escolha do Three Day Physical Activity Recall quanto à equivalência conceitual, semântica e de itens, que resultou na versão brasileira para adolescentes de 10 a 12 anos. Métodos: Foram compilados artigos que continham o instrumento de autorrelato, que tivessem como variáveis de interesse o nível de atividade física e o custo energético. As buscas foram realizadas nas bases de dados BIREME, LILACS, MEDLINE, PubMed, SciELO, Web of Science e SPORTDiscus. Seguiram-se as etapas da adaptação transcultural: dez doutores e 30 membros da população-alvo participaram da avaliação e adequação do constructo à cultura brasileira (equivalência conceitual e de itens) e a versão em português do instrumento seguiu todas as recomendações para equivalência semântica. Resultados: São apresentadas as avaliações dos doutores sobre a versão em português do instrumento, envolvendo questões sobre regionalidade das atividades, dificuldade cognitiva para o autorrelato, instruções, capacidade de mensuração da atividade física e seus domínios. As diferentes versões de tradução e re-tradução do instrumento, com destaque para as modificações solicitadas, assim como a versão final são apresentadas. Conclusões: Tendo sido satisfeitos os pré-requisitos da adaptação transcultural, concluiu-se que o instrumento está adaptado para a população e cultura alvo; entretanto, as qualidades psicométricas, a reprodutibilidade, a validade, o constructo e o critério devem ser testados.

Descritores: atividade motora; metabolismo energético; comparação transcultural; inquéritos e questionários.

\section{ABSTRACT}

Introduction: There are several tools that measure physical activity in Brazil. One of them is the Three Day Physical Activity Recall; however, until now, no tool was subjected to cross-cultural adaptation. Objective: To describe the procedures and criteria for selecting the Three Day Physical Activity Recall on the conceptual, semantic and items equivalence, which resulted in the Brazilian version for adolescents from 10 to 12 years. Methods: Articles have been compiled containing the self-report instrument that had as variables of interest the level of physical activity and energy cost. A survey was conducted in the following databases: BIREME, LILACS, MEDLINE, PubMed, SciELO, Web of Science, and SPORTDiscus. The steps of cultural adaptation came next: 10 doctors and 30 members of the target population participated of the evaluation and adjustment of the construct to the Brazilian culture (conceptual and item equivalence) and the Portuguese version of the instrument followed all recommendations for semantic equivalence. Results: Here we present the evaluations of doctors on the Portuguese version of the instrument, involving questions about regionality of activities, cognitive impairment to self-report, instructions, ability to measure physical activity and their domains. The different versions of the translation and retranslation of the tool, highlighting on the changes requested as well as the final version are presented. Conclusions: Since the prerequisites of cultural adaptation have been met, it was concluded that the tool is adapted for the target population and culture; nevertheless, the psychometric qualities, reproducibility and validity, construct and criterion must be tested.

Keywords: motor activity; energy metabolism; cross-cultural comparison; surveys and questionnaires.

\section{RESUMEN}

Introducción: Existen varios instrumentos que miden la actividad física en Brasil. Uno de ellos es el Three Day Physical Activity Recall. Sin embargo, hasta el momento, ninguna herramienta se sometió a una adaptación transcultural. Objetivo: Describir los procedimientos y criterios para la selección de Three Day Physical Activity Recall relativos a la equivalencia conceptual, semántica y de ítems, lo que resultó en la versión brasileña para adolescentes de 10 a 12 años. Métodos: Se han compilado artículos que contienen el instrumento de autoinforme que tenían como variable de interés el nivel de actividad y los costos energéticos. Las búsquedas se realizaron en las bases de datos BIREME, LILACS, MEDLINE, PubMed, SciELO, Web of Sciencey SPORTDiscus. Luego vinieron las etapas de adaptación transcultural:diez doctores y 30 miembros de la población objetivo participaron de la evaluación y la adecuación del constructo a la cultura brasileña (equivalencia conceptual y de items) y la versión en portugués del instrumento ha seguido todas las recomendaciones para equivalencia semántica. Resultados: Se presentan las evaluaciones de los doctores sobre la versión en portugués del instrumento, que implica cuestiones sobre la regionalidad de las actividades, dificultad cognitiva para el autoinforme, las instrucciones, la capacidad de medición de la actividad física y sus dominios. Se 
presentan las diferentes versiones de traducción y re-traducción del instrumento, destacando los cambios solicitados, así como en la versión final. Conclusiones: Después de haber satisfecho los prerrequisitos de adaptación transcultural se concluyó que el instrumento se adapta a la población y cultura objetivo; no obstante, las cualidades psicométricas, la reproducibilidad, la validez, el constructo y el criterio deben ser probados.

Descriptores: actividad motora; metabolismo energético; comparación transcultural; encuestas y cuestionarios.

\section{INTRODUÇÃO}

Entre 2008 e 2013, estima-se que 3700 artigos, dissertações ou teses estejam relacionados à adaptação transcultural de questionários que mensurem constructos na área de saúde no Brasil. Este número é três vezes maior do que o número de trabalhos sobre este tema no período entre 2002 a 2007. O crescente aumento pode estar relacionado à preocupação na utilização de instrumentos produzidos em outros países, com características culturais específicas, em estudos nacionais. Alguns autores ressaltam que a simples tradução pode trazer consequências sérias quando os estudos buscam estabelecer perfis, padrões, relações ou comparações, pois o entendimento de um constructo no país onde o instrumento se originou, pode ter características bem diferentes em outro país e população-alvo, portanto, podendo trazer considerações distorcidas do fenômeno a ser mensurado ${ }^{1-6}$.

$\mathrm{Na}$ tentativa de minimizar as diferenças culturais e permitir a adequação de instrumentos autorrelato e questionários (surveys) em países diferentes do local de onde se originaram, vários autores têm optado por realizar a adaptação transcultural ${ }^{1,2,7-9}$. A adaptação transcultural não é uma simples tradução, mas se refere a um processo de adequação e sincronização de um instrumento, para o uso em outro país, outra língua e outra cultura ${ }^{1,2,6}$. É um processo que possui diversas etapas e que envolvem tradutores, tradutores bilíngues (nativos na língua origem), doutores e a população-alvo 1,2. O Guidelines for Best Practice in Cross-Cultural Surveys ${ }^{1}$ estabelece parâmetros para o julgamento da necessidade de adaptação transcultural de instrumentos, sendo eles: a cultura, a língua e o país.

Apesar da importância e da necessidade de monitorização do nível de atividade física e custo energético em crianças e adolescentes, quando se reporta a instrumentos que mensuram essas variáveis em trabalhos nacionais, esbarra-se na utilização de muitos instrumentos de origem internacional10-13. A grande maioria não foi adaptada transculturalmente, o que pode ter trazido informações distorcidas dos fenômenos. Cruciani et al. ${ }^{14}$ salientam que na ausência de instrumentos nacionais, é possível adaptar questionários desenvolvidos em outras culturas, porém há a necessidade de utilização de procedimentos consolidados e criteriosos de adaptação transcultural para a criação de uma versão adaptada. E ainda, é imprescindível que as versões adaptadas tenham seus parâmetros psicométricos testados, para que possam apresentar uma boa estimativa das variáveis. Para isso, entende-se que ao adaptar um instrumento, é necessário validar as medidas desse "novo instrumento". Neste sentido, muitos estudos têm sido conduzidos para entender a reprodutibilidade e validade de novos instrumentos, e sua adequação e adaptação a uma nova cultura ${ }^{15}$.

Dumith $^{16}$ em um artigo de revisão sistemática sobre atividade física em adultos no Brasil, encontrou que em 45 estudos nacionais, os instrumentos utilizados para mensurar atividade física e/ou seus domínios, foram produzidos pelos próprios autores (60\%), e os demais instrumentos utilizados são de origem internacional. Identificamos que 51\% dos estudos de atividade física e dispêndio energético envolvendo crianças e adolescentes, utilizaram instrumentos dos próprios autores, e os demais instrumentos eram de origem internacional (Physical Activity Questionnarie for Children (11\%); Three Day Physical Activity Recall (18\%);
International Physical Activity Questionnarie - long and short form (7\%); SelfAdministered Physical Activity Checklist (5\%); Atividade Física Habitual Beacke (4\%); Quarifo (4\%)). Dos instrumentos internacionais, dois passasram pelo processo de adaptação transcultural (International Physical Activity Questionnarie - long and short form e Atividade Física Habitual Beacke), e os demais são utilizados no Brasil por tradução simples.

O Three Day Physical Activity Recall (3DPAR), citado em oito estudos de revisão, no período de 2000 a 2012, não foi submetido ao processo de adaptação transcultural. Este instrumento permite que crianças e adolescentes recordem as atividades realizadas, por autorrelato ou entrevista, durante três dias, sendo dois dias da semana e um dia do final de semana, classificando o nível de atividade física e permitindo o cálculo do dispêndio energético diário.

Portanto, o objetivo do presente estudo foi descrever as etapas dos processos de equivalência conceitual, de itens e semântica da versão brasileira em português do instrumento 3DPAR para uso em crianças e adolescentes de 10 a 12 anos.

\section{MÉTODOS}

O primeiro passo foi encontrar o instrumento de autorrelato que tivesse como variável de desfecho nível de atividade física e custo energético. A partir do interesse dos pesquisadores foram realizadas buscas nas bases de dados BIREME (Biblioteca Virtual em Saúde), LILACS (Literatura LatinoAmericana e do Caribe em Ciências da Saúde - Interface BVS), MEDLINE (Literatura Internacional em Ciências da Saúde - Interface BVS), Pubmed (National Library of Medicine's - NLM), Scielo.org (Scientific Eletronic Library Online), Web of Science (Web of Knowlegde interface) e SPORTDiscus (EBSCO Interface). Foram utilizadas as seguintes palavras-chave e descritores: Child, Children, Childhood, motor activity, Motor activities, Physical Activity, Physical Activities, Sedentary lifestyle, Energy metabolism, Energy Expenditure, Energy Expenditures, Energy Cost, Physical Activity level, Physical inactivity, Brazil, Brazilian. Além das palavras-chave/descritores foram utilizados AND e OR. Os critérios foram estabelecidos de acordo com cada base de dados, mas seguiram a estrutura básica (Figura 1).

Após a identificação do instrumento, o Three Day Physical Activity Recall (3DPAR), foi enviado um e-mail aos autores/criadores solicitando autorização para processo de adaptação transcultural. Após a autorização concedida, foram seguidos os procedimentos propostos por Reichenheim \& Moraes $^{2}$, que são: equivalência conceitual, de itens, semântica, operacional e de mensuração. Porém, o presente estudo apresentará as equivalências conceitual, itens e semântica (Figura 2).

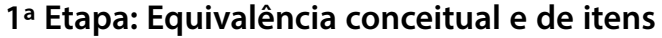

O processo de adaptação iniciou-se com a avaliação da equivalência conceitual. Nessa etapa, foi realizado um julgamento da pertinência dos conceitos e dimensões assimilados pelo instrumento original na cultura da versão traduzida. Foi realizada uma revisão do modelo conceitual que sustenta teoricamente o constructo de interesse, fornecendo a base para a análise do campo de cobertura do instrumento adaptado quanto às características da mensuração. Esta etapa envolveu a discussão com doutores em Educação Física, todos da região sudeste e 


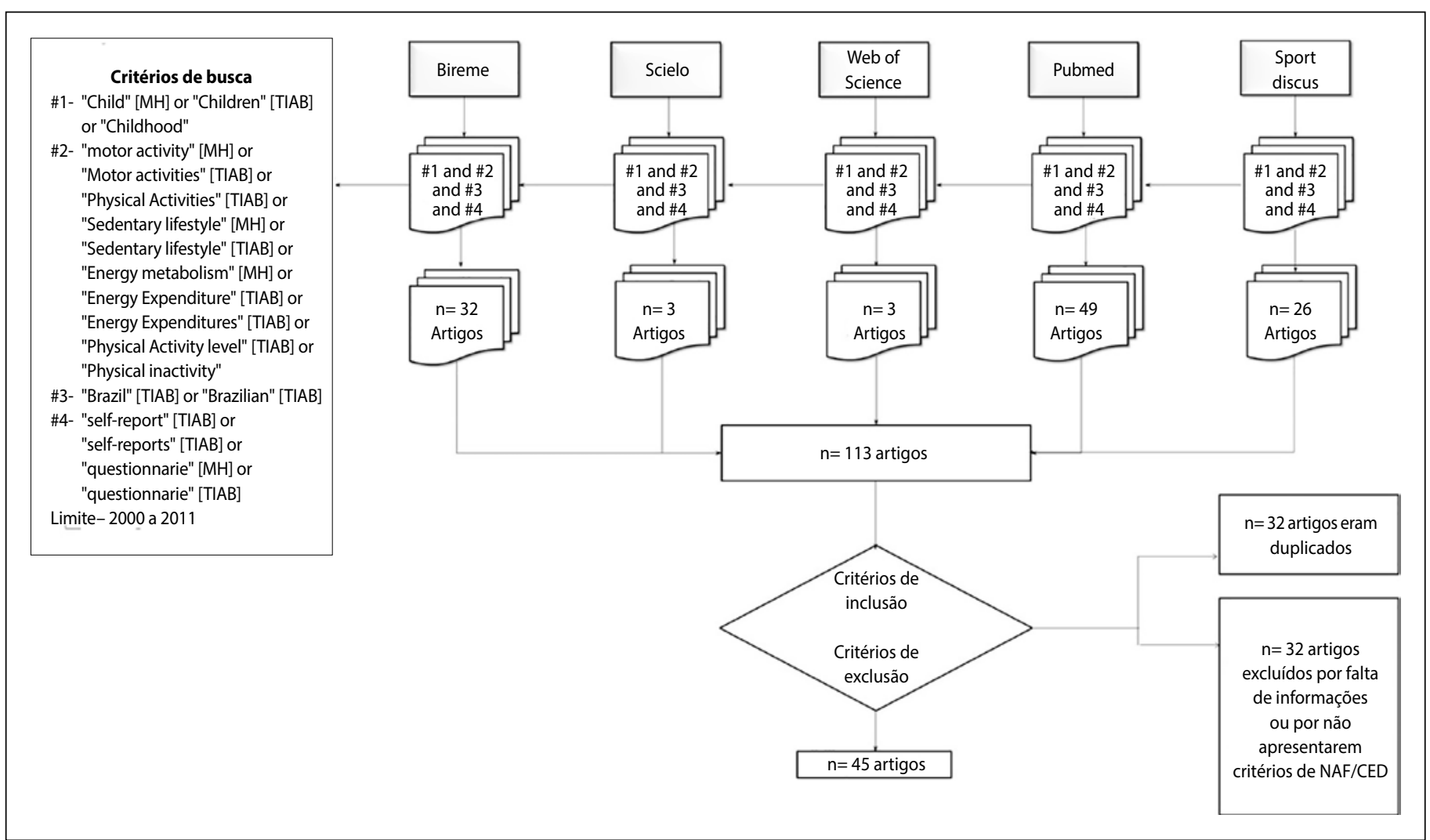

Figura 1. Fluxograma com os critérios de busca para identificação dos artigos e publicações do nível de Atividade Física (NAF) e dispêndio energético diário (DED).

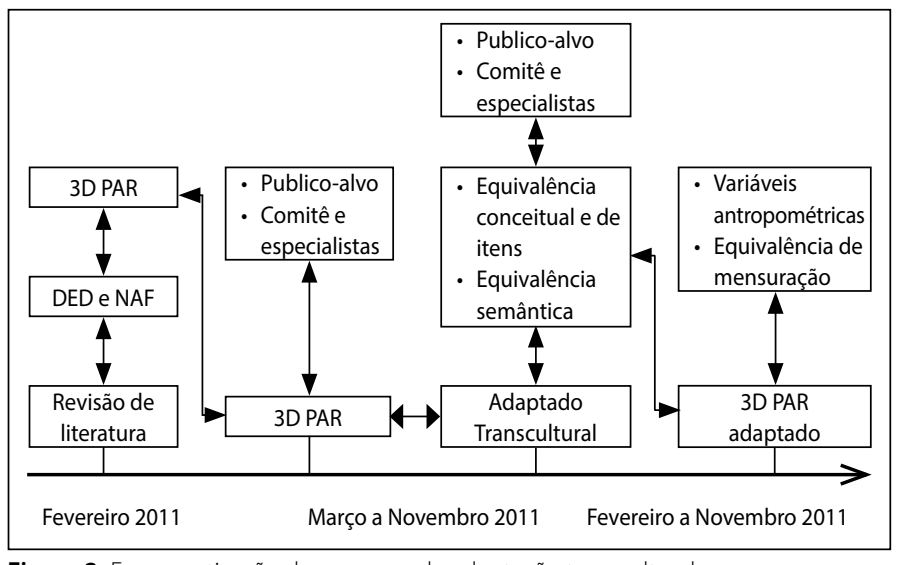

Figura 2. Esquematização do processo de adaptação transcultural.

membros da população-alvo (crianças da mesma faixa etária, porém que não fizeram parte da amostra) envolvida que avaliaram a relevância e a pertinência ao novo contexto ao qual foi adaptado.

Para a realização desta etapa foram convidados 10 doutores na área de Cineantropometria e Educação Física e 30 membros da população-alvo, de uma das escolas municipais da cidade de São João Nepomuceno, Minas Gerais, Brasil. Ambos os grupos foram escolhidos intencionalmente. Os doutores foram identificados por acesso ao curriculum lattes, respeitando-se a regionalidade, titulação e publicações. Após serem comunicados via email e aceitarem participar do estudo, foi solicitado aos mesmos respondessem a um questionário contendo 10 questões fechadas (escala de Likert 1-5) e uma questão aberta com perguntas referentes aos instrumentos e sua relação com o constructo a ser mensurado (atividade física e dispêndio energético).

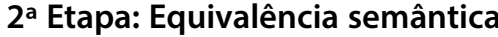

O processo de tradução se iniciou com duas tradutoras brasileiras independentes, juramentadas, que realizaram de modo cego a tradução do inglês para o português do instrumento a ser adaptado (3DPAR).
Primeiramente, realizaram a leitura total do material para ver o contexto, em seguida leram as instruções do doutorando sobre o público-alvo, visto que conhecer o público torna-se importante para que as escolhas lexicais dos tradutores sejam compatíveis com o público-alvo.

Com base nas duas traduções, compuseram-se as duas primeiras versões do questionário adaptado. Após uma reunião de consenso entre as tradutoras, foi gerada a primeira versão do questionário adaptado (V1).

Posteriormente, a V1 foi enviada a dois experientes tradutores bilíngues falantes nativos do inglês para que, de modo cego, retraduzissem para o inglês de forma a comparar com o texto original e detectar possíveis incongruências. Os tradutores não tiveram acesso ao texto-fonte, contexto e ao público-alvo. Concomitantemente, a V1 foi enviada ao mesmo grupo de doutores (apenas cinco participaram dessa etapa) e discutida com os membros da população-alvo, que avaliaram os termos empregados no questionário em questão, para que fosse produzida a versão final do questionário adaptado (V2).

Todos os participantes do estudo assinaram o Termo de Consentimento Livre e Esclarecido, e o trabalho (Projeto no 396/10) foi aprovado pela Comitê de Ética em pesquisa da UFMG (CAAE - 0396.0.203.000-10) .

Para análise dos dados foi utilizado a estatística descritiva e software Excel $^{\oplus}$ for Windows ${ }^{\oplus}$, Brasil.

\section{RESULTADOS}

Nas Tabelas 1 e 2 são apresentados as respostas dos doutores em relação ao questionário que sofreu adaptação transcultural nas etapas 1 e 2, respectivamente.

Na Tabela 3 apresentamos a versão traduzida e a retradução do Three day phsyical activity recall. Além disso, apresentamos os comentários dos tradutores sobre a técnica de equivlência entre os termos. (acesso a Tabela 3. https://www.dropbox.com/s/yn4m7cegyr5h0kw/ Artgo\%20RBME\%20-\%20Tabela\%203.pdf?dl=0).

Na Tabela 4 apresentamos a versão original e adaptada do Recordatório de três dias de atividade física. (acesso a Tabela 4. https://www. 
dropbox.com/s/09gg4z98bz7vp4f/Artgo\%20RBME\%20-\%20Tabela\%204.pdf?dl=0

\section{DISCUSSÃO}

Na Tabela 1, em relação à questão aberta, $60 \%$ dos doutores fizeram comentário sobre a regionalidade das atividades. No questionário original, várias das atividades citadas eram incompatíveis com a cultura local, e talvez até mesmo do Brasil. Alguns doutores (20\%) sugeriram que as crianças e adolescentes teriam dificuldade cognitiva para o autorrelato.

As perguntas da etapa 1 estavam direcionadas sobre a capacidade do instrumento em mensurar/avaliar a atividade física e custo energético no lazer, trabalho, escola, atividades da vida diária e esporte, sobre capacidade do instrumento em mensurar intensidade, se as instruções de utilização estavam claras e de fácil interpretação para o público-alvo. Analisando os valores das médias, moda e mediana dos respondentes, para todas as perguntas, a maioria dos doutores concordou que o instrumento possuía instruções claras e de fácil interpretação, bem como capacidade de mensuração da atividade física e seus domínios (intensidade, duração e frequência semanal).

Segundo os tradutores, os instrumentos não continham linguagem conotativa, predominando o uso denotativo da língua, o que torna bem fácil e direta a tradução. Sendo assim, não foi necessário utilizar nenhuma técnica tradutória específica. A Tabela 3 apresenta as versões de tradução e retradução e os comentários dos tradutores/retradutores/bilíngue, pode-se observar que não foram encontradas muitas diferenças no processo.

Alguns itens foram incorporados de uma das duas versões, na íntegra ou modificados pelo grupo, enquanto outros resultaram da junção das duas versões. Por vezes, o conteúdo desta junção foi modificado para melhor atender aos critérios de equivalência semântica. As reuniões de consenso discutiram a aceitabilidade desta versão na população avaliada e foram propostas novas modificações, o que resultou na elaboração da versão final.

Os tradutores nativos também não encontraram dificuldades, pois na análise das retraduções houve um grau elevado de equivalência de significado referencial e geral. As mínimas discrepâncias em relação ao texto original em inglês não se conformam como diferenças lexicais quanto ao sentido referencial ou geral, sendo os termos divergentes apenas sinônimos dos termos originais. O mesmo ocorreu com a construção das frases. Pequenas diferenças de ordem dos termos não foram consideradas relevantes, pois não alteram o significado geral ou referencial dos termos e frases. Conforme explicita na Tabela 3, o trecho que causou maior discussão entre os tradutores e retradutores, e também foi motivo de discordância entre os doutores foram os termos para discriminar o tipo de respiração associado a intensidade do esforço.

Na Tabela 2, apenas um doutor fez referência a forma de pergunta sobre a intensidade. Este foi um ponto de grande dificuldade do público-alvo, que confundia difícil e muito difícil com a execução do movimento e não com a intensidade. 0 doutor 2 questionou as figuras

Tabela 1. Resposta dos doutores na etapa $1(n=10)$.

\begin{tabular}{|c|c|c|c|c|c|c|c|c|c|c|c|}
\hline & \multicolumn{11}{|c|}{ Perguntas } \\
\hline Doutores & P1 & P2 & P3 & P4 & P5 & P6 & P7 & P8 & P9 & P10 & Comentários \\
\hline 1 & 5 & 5 & 5 & 4 & 3 & 4 & 4 & 4 & 4 & 4 & Regionalidade das atividades \\
\hline 2 & 5 & 2 & 4 & 5 & 5 & 4 & 5 & 3 & 4 & 4 & Regionalidade das atividades \\
\hline 3 & 3 & 3 & 3 & 3 & 3 & 3 & 3 & 4 & 4 & 3 & Regionalidade das atividades \\
\hline 4 & 5 & 3 & 5 & 5 & 5 & 4 & 5 & 5 & 4 & 5 & Regionalidade das atividades \\
\hline 5 & 5 & 4 & 5 & 5 & 5 & 4 & 5 & 4 & 4 & 5 & Regionalidade das atividades \\
\hline 6 & 5 & 5 & 5 & 5 & 5 & 4 & 5 & 4 & 4 & 5 & Regionalidade das atividades \\
\hline 7 & 4 & 4 & 4 & 4 & 5 & 2 & 3 & 2 & 2 & 4 & Sem comentários adicionais \\
\hline 8 & 5 & 4 & 4 & 4 & 5 & 3 & 3 & 2 & 3 & 4 & Sem comentários adicionais \\
\hline 9 & 4 & 2 & 4 & 5 & 4 & 2 & 3 & 3 & 3 & 4 & Cognitivo $x$ instrumento $x$ atividade de trabalho \\
\hline 10 & 4 & 4 & 4 & 5 & 4 & 3 & 3 & 4 & 3 & 4 & Cognitivo $\mathrm{x}$ instrumento $\mathrm{x}$ atividade de trabalho \\
\hline Média & 4,5 & 3,6 & 4,3 & 4,5 & 4,4 & 3,3 & 3,9 & 3,5 & 3,5 & 4,2 & \\
\hline Moda & 5 & 4 & 4 & 5 & 5 & 4 & 3 & 4 & 4 & 4 & \\
\hline Mediana & 5 & 4 & 4 & 5 & 5 & 3,5 & 3,5 & 4 & 4 & 4 & \\
\hline
\end{tabular}

Tabela 2. Respostas dos doutores na etapa $2(n=5)$.

\begin{tabular}{|c|c|c|c|c|c|c|c|c|c|c|c|}
\hline \multirow[b]{2}{*}{ Doutores } & \multicolumn{10}{|c|}{ Perguntas } & \multirow[b]{2}{*}{ Comentários } \\
\hline & P1 & P2 & P3 & P4 & P5 & P6 & P7 & P8 & P9 & P10 & \\
\hline 1 & 4 & 4 & 4 & 4 & 4 & 4 & 4 & 3 & 3 & 2 & Sem comentários \\
\hline 2 & 5 & 5 & 3 & 3 & 4 & 4 & 4 & 4 & 3 & 2 & Figuras moderado; difícil e muito difícil são iguais/ questionário longo \\
\hline 3 & 5 & 2 & 2 & 2 & 5 & 5 & 5 & 4 & 4 & 4 & $\begin{array}{l}\text { Sugestão para crianças: Intensidade LEVE: respiração normal invés } \\
\text { de lenta; MODERADA: respiração alterada, mas você consegue } \\
\text { conversar; DIFICIL: respiração mais rápida, difícil de conversar; } \\
\text { MUITO DIFICIL: respiração muito rápida, não dá para conversar. } \\
\text { No item comer: Snacking não é o lanche como entendemos no } \\
\text { Brasil, está mais para o termo "beliscar fora de hora", ou no máximo } \\
\text { um lanchinho Item } 23 \text { e 24: creio que deslocamento fica melhor que } \\
\text { viagem Item 28: jazz Itens: } 32,38 \text { e } 46 \text { desnecessários } \\
\text { Colocaria o item } 45 \text { inserido nos exemplos do } 47\end{array}$ \\
\hline 4 & 5 & 4 & 3 & 4 & 4 & 4 & 4 & 5 & 5 & 2 & Sem comentários \\
\hline 5 & 4 & 5 & 4 & 4 & 4 & 5 & 3 & 5 & 5 & 3 & Sem comentários \\
\hline Média & 4,8 & 3,8 & 3,0 & 3,3 & 4,3 & 4,3 & 4,3 & 4,0 & 3,8 & 2,5 & \\
\hline Moda & 5 & 4 & 3 & 4 & 4 & 4 & 4 & 4 & 3 & 2 & \\
\hline Mediana & 5 & 4 & 3 & 3,5 & 4 & 4 & 4 & 4 & 4 & 2 & \\
\hline
\end{tabular}


utilizadas no instrumento original, o que foi considerado pertinente. O mesmo comentário foi realizado pela maioria da população alvo. A partir daí, foi solicitado a um designer gráfico que fizesse um desenho, estilo OMNI, para dar a ideia de intensidade crescente. O desenho teve aprovação do público-alvo e dos doutores envolvidos. Além disso, no instrumento adaptado foi acrescentada a palavra esforço a frente dos termos leve, moderado, difícil e muito difícil.

$\mathrm{Na}$ tentativa de melhorar a precisão do relato foram incorporados ao instrumento, para diversas atividades sedentárias, os subitens deitado, sentado e em pé. A justificativa dessas inserções são as diferenças no dispêndio energético entre as diferentes posições, que poderiam introduzir viés no instrumento quando comparado as medidas critério. Além disso, algumas atividades de trabalho foram acrescentadas de acordo com a discussão com a população-alvo.

Outras modificações realizadas no instrumento original foram o acréscimo de festas no item "Depois da escola/tempo livre e hobbies", de higiene pessoal no item "Auto-cuidado (Dormir/Tomar banho) e no item "Escola" foram retiradas as atividades "Educação física escolar" e "Marcha em dia cívil". A justificativa de retirada se deu pelas comemorações cíveis serem realizadas fora da escola, enquanto a retirada da educação física escolar foi devido as aulas serem direcionadas a desportos ou atividades formais.
Para o item "Trabalho", utilizando o compendium ${ }^{17}$ de atividade física para criança e adolescente, subdividimos as atividades de casa em atividades que tiveram seu dispêndio energético diário definido para a população-alvo.

Atividades como surfing, futebol americano, softball/baseball, peso livre, luta-livre, foram retiradas por sugestão da população-alvo que discordou que tais atividades faziam parte do seu dia-a-dia. Diante dessas alterações, a listagem ficou com 62 atividades.

\section{CONCLUSÕES}

Todos os pré-requisitos da adaptação transcultural para o recordatório de três dias de atividade física foram executados, e, portanto o instrumento encontra-se adaptado para a população e cultura alvo. Porém, é necessário que o "novo" instrumento tenha suas qualidades psicométricas (reprodutibilidade, validade constructo e critério) para que possamos conhecer a qualidade da medida de atividade física e dispêndio energético.

Todos os autores declararam não haver qualquer potencial conflito de interesses referente a este artigo.

CONTRIBUIÇÕES DOS AUTORES: Cada autor contribuiu individual e significativamente para o desenvolvimento do manuscrito. VOD (0000-0003-0577-9204)* autor principal, foi o responsável por toda elaboração do artigo. ACS (0000-0002-4327-9190)* participou na busca em bases dados e levantamento bibliográfico dos instrumentos. PRA (0000-0002-4327-9190)* e JRPL (0000-0003-0073-8673)* participaram na definição dos métodos. JMV (0000-0003-1594-4429)*, RG (0000-0001-6089-8375)* e FZW (0000-0003-1966-8820)* participaram na definição dos métodos e colaboração na discussão dos resultados. JAL (0000-0002-0581-3217)* foi o orientador do trabalho. *ORCID (Open Researcher and Contributor ID).

\section{REFERÊNCIAS}

1. Survey Research Center. (2016). Guidelines for Best Practice in Cross-Cultural Surveys. Ann Arbor, Ml: Survey Research Center, Institute for Social Research, University of Michigan. [acesso em 2016 out 16]. from http://www.ccsg.isr.umich.edu/

2. Reichenheim ME, Moraes CL. Operationalizing the cross-cultural adaptation of epidemological measurement instruments. Rev Saude Publica. 2007;41(4):665-73.

3. Beaton DE, Bombardier C, Guillemin F, Ferraz MB. Guidelines for the process of cross-cultural adaptation of self-report measures. Spine. 2000;25(24):3186-91.

4. Geisinger KG. Cross-cultural normative assessment: Translation and adaptation issues influencing the normative interpretation of assessment instruments. Psychol Assess. 1994;6(4):304-12.

5. Guillemin F, Bombardier C, Beaton D. Cross-cultural adaptation of health-related quality of life measures: literature review and proposed guidelines. J Clin Epidemiol. 1993;46(12):1417-32.

6. Behling O, Law KS. Translating Questionnaires and other Research Instruments. 133 ed. Thousand Oaks: Sage Publications; 2000

7. Victor JF, Ximenes LB, Almeida PC. Cross-cultural adaptation of the Exercise Benefits/Barriers Scale (EBBS) for application in elderly Brazilians: preliminary version]. Cad Saude Publica. 2008;24(12):2852-60.

8. Oyeyemi AL, Oyeyemi AY, Adegoke BO, Oyetoke FO, Aliyu HN, Aliyu SU, et al. The Short Internationa Physical Activity Questionnaire: cross-cultural adaptation, validation and reliability of the Hausa language version in Nigeria. BMC Med Res Methodol. 2011;11:156
9. Matsuzaki M, Haruna M, Ota E, Yeo S, Murayama R, Murashima S. Translation and cross-cultural adaptation of the Pregnancy Physical Activity Questionnaire (PPAQ) to Japanese. Bioscience Trends. 2010;4(4):170-7.

10. Alves JGB, Siqueira PP, Figueiroa JN. Overweight and physical inactivity in children living in favelas in the metropolitan region of Recife, Brazil. J Pediatr (Rio J). 2009;85(1):67-71.

11. Farias Júnior JC. Associação entre prevalência de inatividade física e indicadores de condição socioeconômica em adolescentes. Rev Bras Med Esporte. 2008;14(2):109-14.

12. Silva KS, Vasques DG, Martins CO, Williams LA, Lopes AS. Active commuting: prevalence, barriers, and associated variables. J Phys Act Health. 2011 Aug;8(6):750-7.

13. Frainer DES, Silva MCM, Santana MLP, Santos NS, Oliveira LPM, Barreto ML, et al. Prevalência e fatores associados ao excesso de peso em adolescentes de Salvador, Bahia, Brasil. RevBras Med Esporte. 2011;17(2):102-6

14. Cruciani F, Adami F, Assunção NA, Bergamaschi DP. Equivalência conceitual, de itens e semântica do Physical Activity Checklist Interview (PACl). Cad Saúde Pública. 2011;27(1):19-34.

15. Kohl HW, Fulton JE, Caspersen CJ. Assessment of physical activity among children and adolescents: a review and synthesis. Preventive medicine. 2000;31(2):S54-76.

16. Dumith SC. Physical activity in Brazil: a systematic review. Cad Saúde Pública. 2009;25( Suppl 3):S415-26.

17. Ridley K, Ainsworth BE, Olds TS. Development of a compendium of energy expenditures for youth. Int J Behav Nutr Phys Act. 2008;5:45 